\title{
A Path Loss Model for Vehicle-to-Vehicle Visible Light Communications
}

\author{
Hossien B. Eldeeb*, Farshad Miramirkhani ${ }^{\dagger}$, and Murat Uysal*, \\ *Department of Electrical and Electronics Engineering, Ozyegin University, Istanbul, Turkey, 34794. \\ ${ }^{\dagger}$ Department of Electrical and Electronics Engineering, Isik University, Istanbul, Turkey, 34980. \\ (e-mails: hossien.eldeeb@ozu.edu.tr, farshad.miramirkhani@isikun.edu.tr, and murat.uysal@ozyegin.edu.tr)
}

\begin{abstract}
The increasing adoption of LEDs in exterior automotive lighting makes visible light communication (VLC) a natural solution for vehicular networking. In this paper, we consider a vehicle-to-vehicle link and propose a path loss expression as a function of distance and different weather conditions. We conduct ray tracing simulations and verify the accuracy of proposed expression. We further use this expression to derive the achievable transmission distance for a targeted data rate while satisfying a given value of bit error rate. Numerical results are presented to demonstrate the achievable distances for single and dual photodetector deployment cases.
\end{abstract}

Index Terms - Vehicular visible light communication, channel modeling, vehicle-to-vehicle communication

\section{INTRODUCTION}

In the last decade or so, there have been extensive research efforts in the context of Intelligent Transportation Systems (ITSs) to improve road safety, traffic flow, and passenger comfort [1]. As a milestone of future generation ITSs, fully autonomous vehicles are being considered by major automakers as well as Google, Uber, and Tesla [2]. One of the keys enabling technologies for such advanced transport solutions is vehicular connectivity in the form of vehicle-to-vehicle $(\mathrm{V} 2 \mathrm{~V})$, vehicle-to-infrastructure (V2I), vehicle-to-pedestrian (V2P), commonly referred to as V2X. Dedicated short-range communications (DSRC) and long-term evolution vehicle (LTE-V) were proposed as vehicular connectivity solutions [3]. As such, DSRC was already deployed by some auto manufacturers [4] while early LTE-V prototypes are also available [5].

As an alternative vehicular connectivity solution, visible light communication (VLC) was proposed based on the dual use of LEDs as wireless transmitters [6]. The increasing adoption of LEDs in automotive lighting, including brake lights, headlamps, taillights and turn signals makes VLC a natural solution for vehicular networking. The existing literature on VLC is mainly geared towards indoor applications while there are relatively less efforts on vehicular VLC systems [7]. Some works in this area assume the use of camera (which is already found as a built-in feature in many vehicles) as the receiver.

The work of Hossien B. Eldeeb was supported by the European Horizon 2020 MSC ITN (VISION) under Grant 764461. The work of M. Uysal was supported by the Turkish Scientific and Research Council (TUBITAK) under Grant 215E311.
However, such systems are limited to low data rates due to camera frame rate. The use of photodetectors is required to enable high data rates. These photodetectors can be placed in different parts of the car to ensure an omnidirectional coverage.

An important research topic in vehicular VLC systems is channel modeling which is critical for link budget calculations and an optimized system design. Earlier works on vehicular VLC channel modeling mainly built upon some ideal, yet unrealistic assumptions. For example, some works [8]-[10] considered the Lambertian illumination pattern which might be valid for indoor light sources, but do not capture the asymmetrical intensity distribution of vehicle headlights. Another concern is the effect of road reflectance which has captured only some recent attention [11], [12].

Furthermore, adverse weather conditions might strongly affect the vehicular channel. In an effort to quantify the effect of rain and fog, we investigated a V2V link with a high beam headlamp acting as the transmitter and a single photodetector as the receiver in a previous paper [13]. Specifically, based on ray tracing, we obtained the channel impulse response (CIR) between two cars travelling in the same lane and proposed a path loss expression as a linear function of transmission range applicable for short trailing distances.

In this paper, we investigate $\mathrm{V} 2 \mathrm{~V}$ channel modeling for larger transmission ranges. Our ray tracing simulation results demonstrate that the linear model in [13] works only for ranges less than around 20 meters. To address this, we propose a new path loss expression which takes the form of a negative exponential function and provides an excellent match to simulation results for larger transmission ranges and under different weather conditions. Then, we use this expression to derive the achievable transmission distance for a targeted data rate while satisfying a given value of bit error rate (BER). We also explore the benefits of using dual photodetectors conveniently located under taillights as receivers and quantify improvements over the single photodetector case. The remainder of this paper is organized as follows. In Section II, we describe our channel modeling approach. In Section III, we propose a closed-form path loss expression as function of distance and channel parameters. In Section IV, we derive an expression for the achievable transmission distance to attain a targeted data rate. In Section V, we present numerical results and finally conclude in Section VI. 


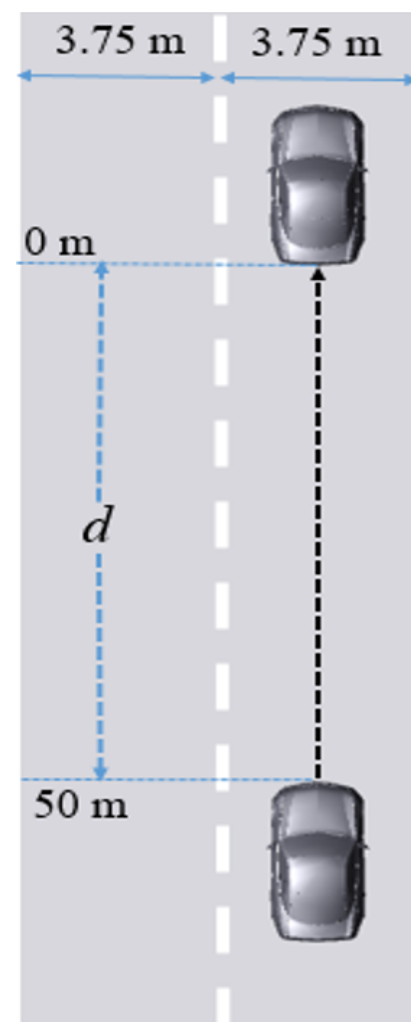

Fig. 1. V2V scenario under consideration

\section{Channel Modeling Approach}

We use non-sequential ray tracing tools of optical design software Zemax ${ }^{\circledR}$ for channel modeling. This modeling approach was originally used in the context of indoor VLC channels [14] and later applied to V2V VLC channels in [13]. As illustrated in Fig. 1, we consider a V2V scenario in a two-lane highway road with a lane width of $3.75 \mathrm{~m}$ [15]. We assume that two cars are located at the center of the same lane and separated with an inter-vehicle distance of $d$. The three dimensional simulation environment is constructed in Zemax ${ }^{\circledR}$. Following the specifications of [6], we assume R2 type asphalt road which has mixed diffuse and specular reflection nature. We adopt Mie scattering to model clear, rainy and foggy weather conditions [16].

As illustrated in Fig. 2, we assume that cars are blackcolored and modeled as CAD objects with dimensions of 4.6 $\mathrm{m} \times 1.8 \mathrm{~m} \times 1.3 \mathrm{~m}$ following Audi A5 Coupe specifications [17]. Headlamps of the first car serve as wireless transmitters. They are designed to provide an adequate road illumination without causing any glare for other road users, therefore have asymmetrical intensity distributions patterns. At the receiver side, we consider two cases: Case A) We assume a single photodetector (PD1) located at the center of the back of the car similar to [13], Case B) We assume the deployment of two photodetectors (PD2 and PD3) installed under taillights as shown in Fig. 2. In Zemax ${ }^{\circledR}$ simulation, a rectangular surface with specified dimensions is used as a receiving element. All simulation parameters are provided in Table 1.
Non-sequential ray tracing is then used to obtain the optical power, the path length, and the propagation delay of each ray emitted from the headlamps and reaching to the $\operatorname{PD}(\mathrm{s})$. Let $N_{P}$ denotes the number of PDs. Obviously, $N_{P}$ is equal to 1 and 2 respectively for Case A and Case B described above. For a given transmission distance, let $P_{i j}$ and $\tau_{i j}$ respectively denote the power and the propagation delay of the $i^{\text {th }}$ ray received by the $j^{t h} \mathrm{PD}, j=1, \cdots, N_{P}$. The CIR at the $j^{t h}$ PD can be therefore written as

$$
h_{j}(t)=\sum_{i=1}^{N_{j}} P_{i j} \delta\left(t-\tau_{i j}\right)
$$

where $\delta$ is the Dirac delta function and $N_{j}$ is the number of rays received by the $j^{\text {th }} \mathrm{PD}$.

In simulations, the total optical power of transmitters is assumed to be unity. The CIR can be then scaled for the given value of transmit power $P_{t}$. The received power can be calculated as $P_{r}=P_{t} \sum_{j=1}^{N_{P}} H_{j}$ where $H_{j}=\int_{0}^{\infty} h_{j}(t) d t$ is the corresponding channel DC gain.

\section{Proposed PATH Loss EXPRESSION}

In this section, we propose a closed-form path loss expression for vehicular V2V link and verify it through simulation results. The received optical power at a distance $d$ can be written as

$$
P_{r}=P_{t} G_{e} G_{a t t}
$$

where $G_{a t t}$ represents the atmospheric attenuation and changes according to weather conditions. According to BeerLambert formula [16], it can be expressed as $G_{a t t}=$ $\exp (-c d)$ where $c$ is the extinction coefficient. For clear, rainy and foggy weathers, the extinction coefficient respectively takes the values of $c=1.5 \times 10^{-5}, 0.9 \times 10^{-3}$, and 0.078 [18].

$G_{e}$ is the geometrical loss due to spreading of the emitted optical beam along the distance between the transmitter and the receiver. To take into account the asymmetrical pattern of high-beam source, we propose the use of

$$
G_{e}=A\left(\frac{1}{d^{2}}\right)^{B}
$$

where $A$ represents the geometrical loss value at a reference distance specified as $d_{0}$ and $B$ is the decaying factor. Replacing $G_{a t t}$ and $G_{e}$ in (2), we can express the path loss model as

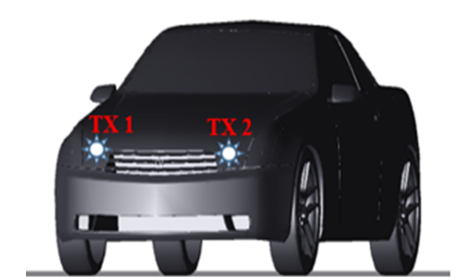

(a)

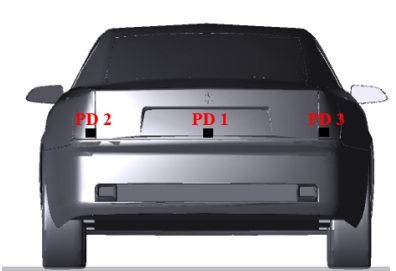

(b)
Fig. 2. Location of high-beam headlamps (transmitters) and photodetectors (receivers) on the vehicles 
TABLE I

SIMULATION PARAMETERS

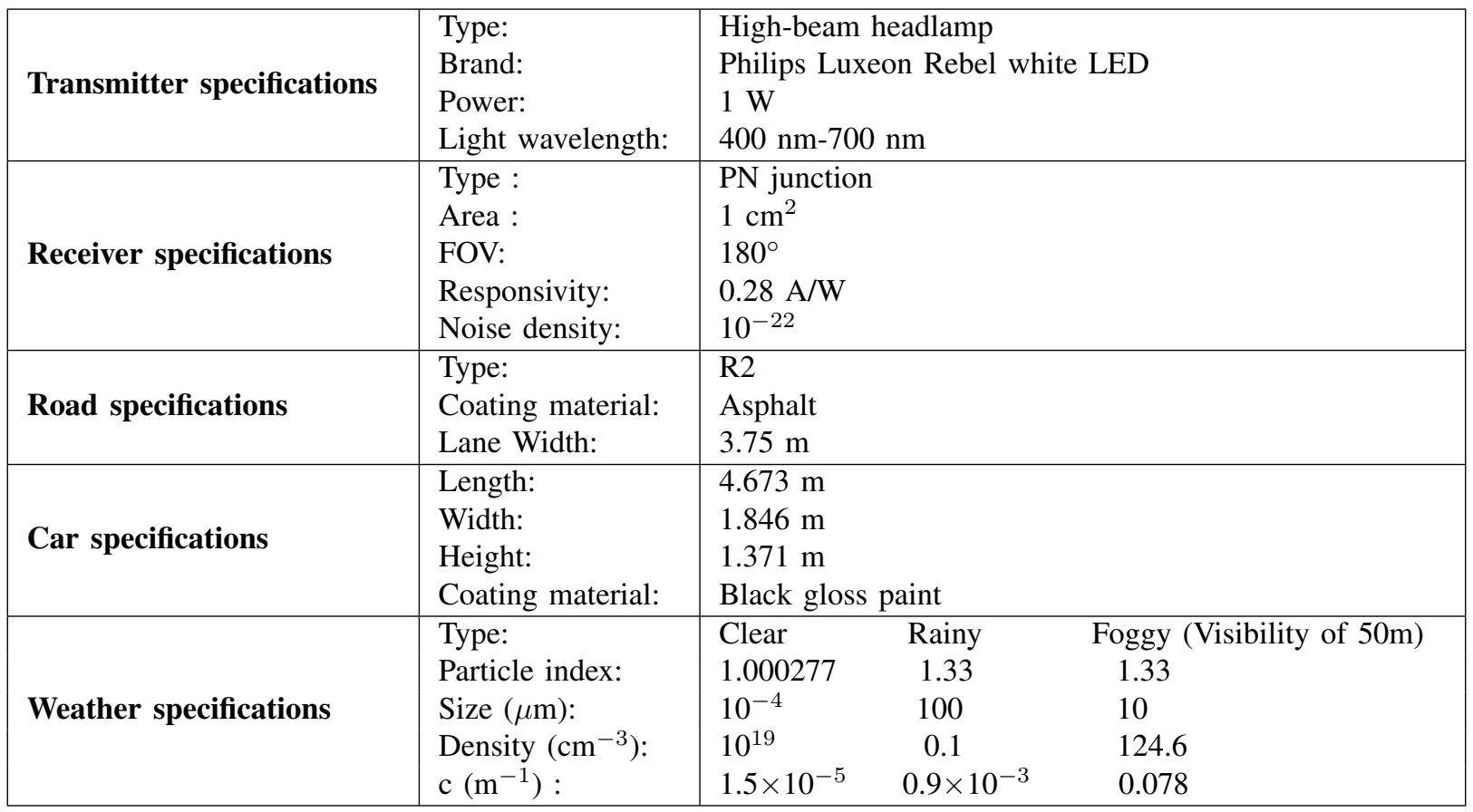

$$
P_{r}=P_{t} \underbrace{A d^{-2 B} \exp (-c d)}_{\rho}
$$

where $\rho$ is the optical channel coefficient.

In order to determine $A$ and $B$, we determine the received optical power at a reference distance $d_{0}=10 \mathrm{~m}$ via Zemax simulations explained in the previous section. Let $P_{0}$ denotes the received optical power at $d_{0}=10 \mathrm{~m}$. We can then determine $A=P_{0} d_{0}{ }^{2 B} \exp \left(c d_{0}\right) / P_{t}$. Through our simulation results, the value of $B$ is determined to be 0.87 for clear and rainy weathers while in case of foggy weather, it is determined to be 0.7 .

As a benchmark, we consider the linear path loss model of [13] which is given by

$$
P_{r}=P_{t}(\alpha d+\beta)
$$

where the values of $\alpha$ and $\beta$ are given as $(\alpha, \beta)=(-0.44$, $-40.93),(-0.46,-40.90)$, and $(-0.61,-40.46)$, respectively, for clear, rainy and foggy weathers.

\section{Achievable Transmission Distance}

In this section, we derive the achievable transmission distance for a targeted data rate while satisfying a given value of BER. Under the assumption of L-ary pulse amplitude modulation (PAM), the BER can be approximated by [19]

$$
B E R \approx \frac{2(L-1)}{L \log _{2}(L)} Q\left(\frac{1}{L-1} \sqrt{\frac{\left(\eta \rho P_{t}\right)^{2} \log _{2} L}{N_{0} R}}\right)
$$

where $L$ is constellation size, $\eta$ represents the responsivity of PD, $R$ is the data rate, and $N_{0}$ is the noise power spectral density. By rearranging (6), we get

$$
R \approx \frac{\left(\eta P_{t} \rho\right)^{2} \log _{2} L}{N_{0}(L-1)^{2} Q^{-1}\left(\frac{B E R L \log _{2} L}{2(L-1)}\right)^{2}}
$$

Let $R_{\text {target }}$ and $B E R_{\text {th }}$ respectively denote the targeted data rate and constraint BER. Solving (7) for $\rho$, we obtain

$$
\rho \approx\left((L-1) \sqrt{\frac{N_{0} R_{\text {target }}}{\left(\eta P_{t}\right)^{2} \log _{2} L}}\right) Q^{-1}\left(\frac{B E R_{\mathrm{th}} L \log _{2} L}{2(L-1)}\right)
$$

After some straightforward mathematical manipulations on $\rho=A d^{-2 B} \exp (-c d)$, see (4), we can obtain $d$ as

$$
d=\frac{2 B}{c} \mathrm{~W}\left(\frac{c A^{\frac{1}{2 B}}}{2 B \rho^{\frac{1}{2 B}}}\right)
$$

where W(.) denotes Lambert-W function. By replacing (8) in (9), the achievable transmission distance for the given values of $R_{\mathrm{target}}$ and $B E R_{\mathrm{th}}$ is obtained as (10).

\section{Simulation Results and Discussion}

In this section, we first confirm the accuracy of the proposed path loss expression through simulations. Then, we present numerical results for the achievable transmission distance under different weather types to achieve specified values of $R_{\mathrm{target}}$ and $B E R_{\mathrm{th}}$. Simulation parameters are 


$$
d=\frac{2 B}{c} \mathrm{~W}\left(\frac{c}{2 B}\left(\frac{A_{0} \eta P_{t} \sqrt{\log _{2} L}}{(L-1) \sqrt{N_{0} R_{\text {target }} Q^{-1}\left(\frac{B E R_{\mathrm{th}} L \log _{2} L}{2(L-1)}\right)}}\right)^{\frac{1}{2 B}}\right)
$$

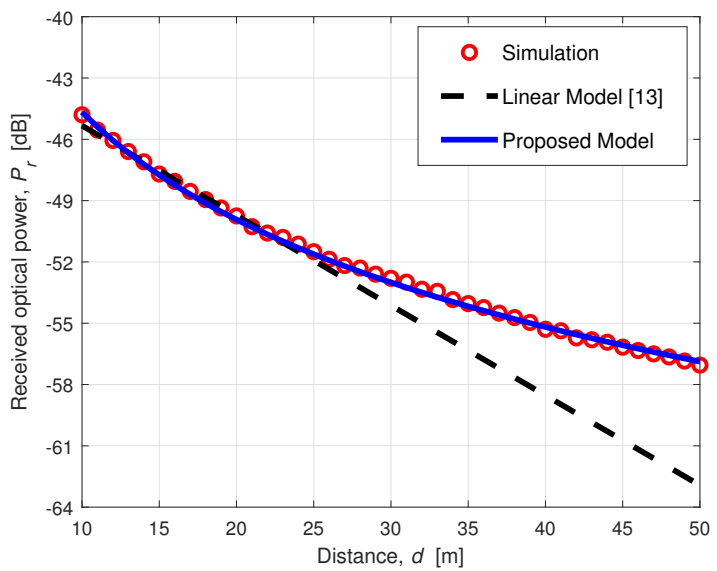

(a)

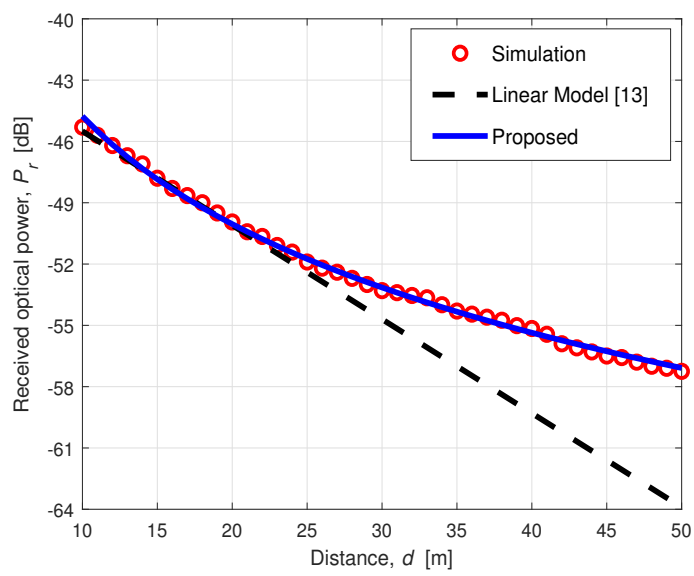

(b)

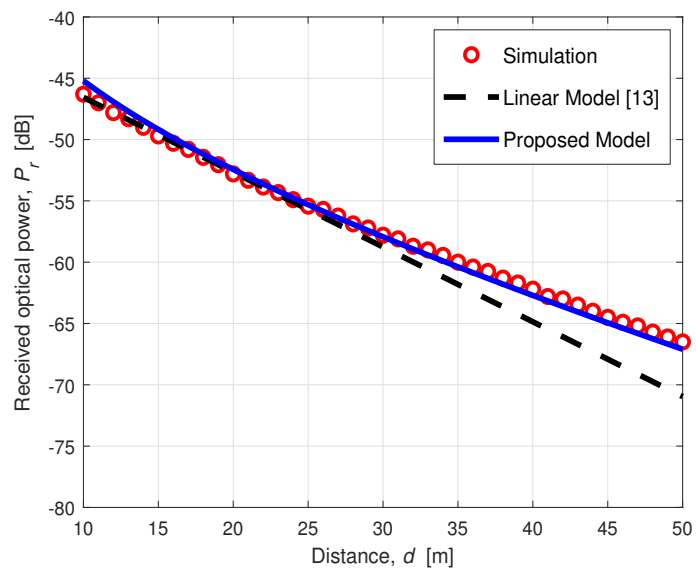

(c)

Fig. 3. Received optical power versus distance assuming single PD deployment (i.e., Case A) for (a) clear weather (b) rainy weather (c) foggy weather

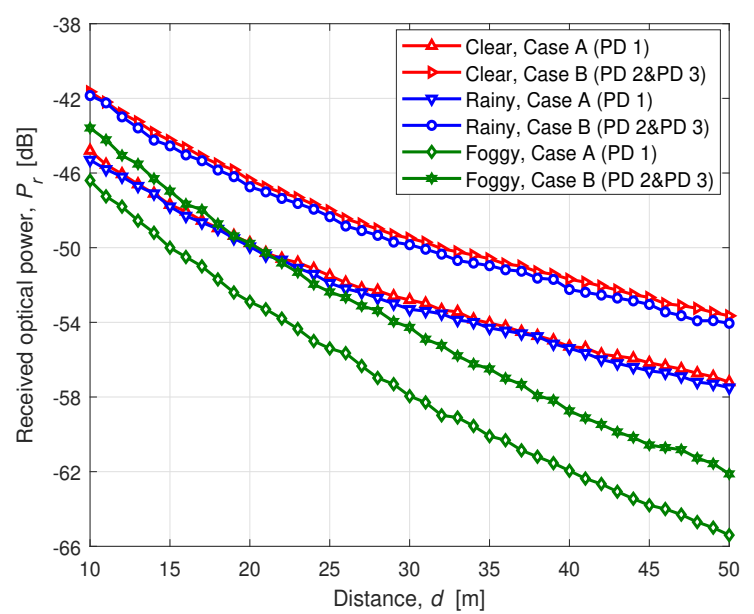

Fig. 4. Performance improvements due to dual photodetector deployment

presented in Table I.

In Fig.3, we present the received power based on the path loss expression in (4) assuming single PD deployment (i.e., Case A) and considering clear, rainy and foggy weather conditions. As benchmarks, we provide simulation results and the linear path loss model in (5). It is observed that the linear model can match simulation results for only short transmission distances and deviates for distances larger than $24 \mathrm{~m}$. On the other hand, the proposed expression in (4) provides a very good match to simulation results for a wider range. Comparison of Figs. 3.a, 3.b. and 3.c further reveals that the rain has negligible effect on VLC link while fog introduces significant degradation. As an example, consider $d=30 \mathrm{~m}$. The received power in clear weather condition is $-52.7 \mathrm{~dB}$ for the normalized unit power transmission. This reduces to -53.3 $\mathrm{dB}$ for rainy condition indicating a mere $0.6 \mathrm{~dB}$ degradation with respect to clear weather.It can be readily verified from Fig. 3.c that this further reduces to $-57.9 \mathrm{~dB}$ for foggy weather and a degradation of $5.1 \mathrm{~dB}$ is observed.

In Fig. 4, we consider Case B where two photodetectors (PD2 and PD3) are deployed and quantify the improvement over the single photodetector case. For clear weather conditions, the received power is $-49.5 \mathrm{~dB}$ at $d=30 \mathrm{~m}$ indicating $3.2 \mathrm{~dB}$ improvement over the single photodetector (PD1) case. Similarly, in rainy and foggy conditions, improvements of 3.5 $\mathrm{dB}$ and $3.63 \mathrm{~dB}$ are respectively observed.

In Fig. 5, we present the achievable transmission distance versus the data rate. We assume $B E R_{\mathrm{th}}=10^{-6}$ and 32-PAM. Assume a target data rate of $R_{\text {target }}=10 \mathrm{Mb} / \mathrm{s}$. In Fig. 5.a for single PD deployment, it is observed that the achievable 


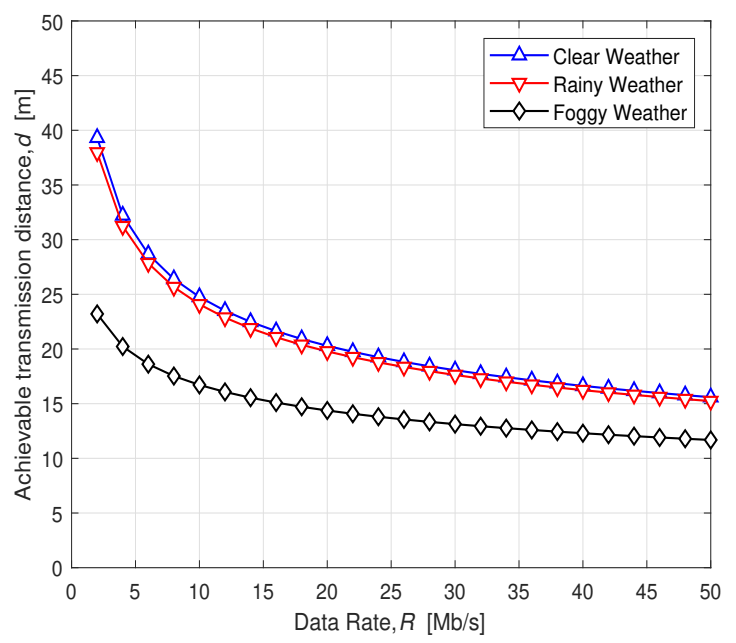

(a)

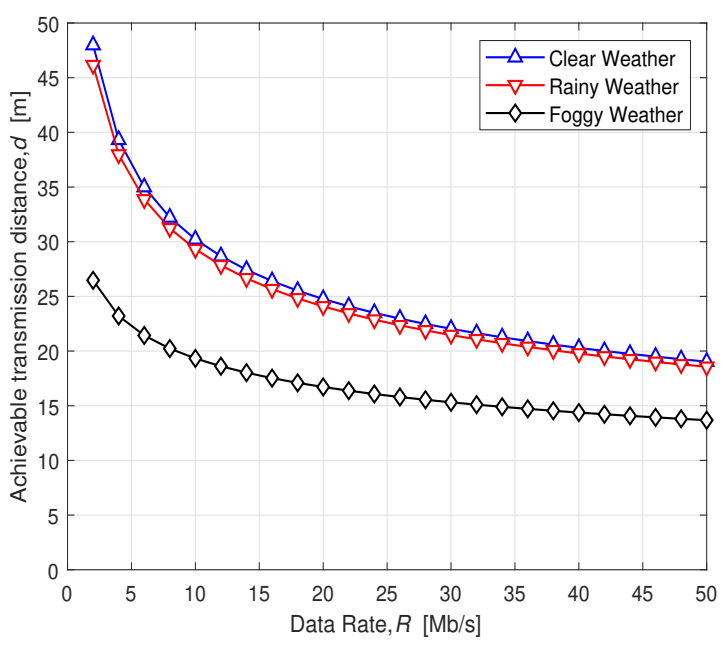

(b)

Fig. 5. Achievable transmission distance versus the data rate for (a) Case A (PD1) (b) Case B (PD2 and PD3)

transmission distance is $25 \mathrm{~m}$ for this data rate. This reduces to $24 \mathrm{~m}$ and $16.5 \mathrm{~m}$ respectively for rainy and foggy weathers. It can be readily verified from Fig. 5.b that using two PDs will increase the achievable transmission distance. The achievable transmission distances are obtained as $30.5 \mathrm{~m}, 29.3 \mathrm{~m}$, and $19.5 \mathrm{~m}$, respectively, for clear, rainy, and foggy weathers.

\section{CONCLUSIONS}

In this paper, we have proposed a path loss expression for V2V VLC link which takes the form of a negative exponential function and provides an excellent match to ray tracing simulation results under different weather conditions. Based on this expression, we have determined the achievable transmission distance for a targeted data rate while satisfying a given value of bit error rate. We have presented numerical results for clear, rainy and foggy weather conditions and quantified the degradation due to adverse weather conditions. We have further quantified performance improvements through dual photodetector use over the single photodetector case.

\section{REFERENCES}

[1] A. Perallos, U. Hernandez-Jayo, I. J. G. Zuazola, and E. Onieva, Intelligent Transport Systems: Technologies and Applications. John Wiley \& Sons, 2015.

[2] J.-R. Xue, J.-W. Fang, and P. Zhang, "A survey of scene understanding by event reasoning in autonomous driving," International Journal of Automation and Computing, vol. 15, no. 3, pp. 249-266, 2018.

[3] G. Naik, J. Liu, and J. J. Park, "Coexistence of wireless technologies in the $5 \mathrm{gHz}$ bands: A survey of existing solutions and a roadmap for future research," IEEE Commun. Surveys Tuts., vol. 20, no. 3, pp. 1777-1798, thirdquarter 2018.

[4] "Toyota commits big to DSRC," https://www.adandp.media/blog/post/ toyota-commits-big-to-dsrc, [Accessed: May 24, 2019].

[5] "Cellular v2x: Continental successfully conducts field trials in china," https://www.continental-corporation.com/en/press/press-releases/201712-18-cellular-v2x-116994, [Accessed: May 24, 2019].

[6] M. Uysal, Z. Ghassemlooy, A. Bekkali, A. Kadri, and H. Menouar, "Visible light communication for vehicular networking: Performance study of a v2v system using a measured headlamp beam pattern model," IEEE Veh. Technol. Mag., vol. 10, no. 4, pp. 45-53, Dec 2015.

[7] A. Cilean and M. Dimian, "Current challenges for visible light communications usage in vehicle applications: A survey," IEEE Commun. Surveys Tuts., vol. 19, no. 4, pp. 2681-2703, Fourthquarter 2017.

[8] N. Kumar, D. Terra, N. Lourenco, L. N. Alves, and R. L. Aguiar, "Visible light communication for intelligent transportation in road safety applications," in 2011 IEEE 7th Int. Wireless Commun. and Mobile Computing Conference, 2011, pp. 1513-1518.

[9] T. Lang, Z. Li, A. Wang, and G. Chen, "Hemispherical lens featured beehive structure receiver on vehicular massive mimo visible light communication system," in Proc. Int. Conf. Internet of Vehicles, 2015 , pp. 469-477.

[10] Y. Bao, Y. Wang, J. Yu, and J. Shen, "A visible light communication based vehicle collision avoiding system," in 15th Int. Conf. Optical Communications and Networks (ICOCN), Sep. 2016, pp. 1-3.

[11] B. Turan, S. Ucar, S. C. Ergen, and O. Ozkasap, "Dual channel visible light communications for enhanced vehicular connectivity," in 2015 IEEE Veh. Networking Conference (VNC), Dec 2015, pp. 84-87.

[12] B. Turan, G. Gurbilek, A. Uyrus, and S. C. Ergen, "Vehicular vlc frequency domain channel sounding and characterization," in 2018 IEEE Veh. Networking Conference (VNC), Dec 2018, pp. 1-8.

[13] M. Elamassie, M. Karbalayghareh, F. Miramirkhani, R. C. Kizilirmak, and M. Uysal, "Effect of fog and rain on the performance of vehicular visible light communications," in 2018 IEEE 87th Veh. Technology Conference (VTC Spring), June 2018, pp. 1-6.

[14] F. Miramirkhani and M. Uysal, "Channel modeling and characterization for visible light communications," IEEE Photon. J., vol. 7, no. 6, pp. 1-16, Dec 2015

[15] P. Colonna, P. Intini, N. Berloco, and V. Ranieri, "Integrated americaneuropean protocol for safety interventions on existing two-lane rural roads," European transport research review, vol. 10, no. 1, p. 5, 2018

[16] M. Uysal, C. Capsoni, Z. Ghassemlooy, A. Boucouvalas, and E. Udvary, Optical wireless communications: an emerging technology. Springer, 2016.

[17] Audi A5., https://www.audiusa.com/models/audi-a5-coupe, [Accessed: May 24, 2019].

[18] H. Willebrand and B. S. Ghuman, Free space optics: enabling optical connectivity in today's networks. SAMS publishing, 2002.

[19] S. Hranilovic, Wireless optical communication systems. New York, NY, USA: Springer-Verlag, 2005. 